\title{
The Effects of Leukocyte-Poor Platelet Rich Plasma for the Symptomatic Knee Osteoarthritis: A Single Centre Experience
}

\author{
Andrea Farneti ${ }^{1 *}$, Michela Giustozzi ${ }^{2}$, Marta Micheli ${ }^{3}$, Enrico \\ Sebastiani ${ }^{1}$, Michele Berloco ${ }^{1}$, Luca Pennacchi ${ }^{1}$, Carlo Farneti ${ }^{1}$ and \\ Fabio Perrone ${ }^{1}$ \\ ${ }^{1}$ Orthopaedics and Traumatology Department, San Giovanni Battista Hospital, \\ Foligno, Italy \\ ${ }^{2}$ Internal Vascular and Emergency Medicine and Stroke Unit, University of Perugia, \\ Perugia, Italy \\ ${ }^{3}$ Transfusion Medicine Department San Giovanni Battista Hospital, Foligno, Italy \\ *Corresponding Author: Andrea Farneti, Orthopaedics and Traumatology \\ Department, San Giovanni Battista Hospital, Foligno, Italy.
}

Received: November 05, 2020

Published: November 27, 2020

(C) All rights are reserved by Andrea

Farneti., et al.

\begin{abstract}
Purpose: Platelet-rich plasma (PRP) is routinely used in the symptomatic treatment of knee osteoarthritis (OA) despite limited evidence. The aim of this study was to evaluate the efficacy of injective protocol of leukocyte-poor-PRP for the symptomatic treatment of knee OA.

Methods: A retrospective study of 213 patients (a total of 326 knees treated) with symptomatic knee OA were analysed; each patient underwent a cycle of three infiltrations with intra-articular PRP and was re-evaluated at 3, 6 and 12 months with clinical examination or phone contact. The Visual Analog Scale (VAS) and International Knee Documentation Committee Subjective Knee Form (IKDC) were used to assess efficacy.

Results: Mean age was $68.1 \pm 9.7$ years and 59.6\% were female. Mean VAS score before treatment was $6.5 \pm 1.4$ (range from 2 to 10 ), $1.6 \pm 1.6$ at 3 months, $1.4 \pm 1.5$ at 6 months and $1.7 \pm 1.7$ at 12 months. A significant reduction was observed between the VAS score pre-treatment and the VAS score at 3 months ( $p<0.001), 6$ months $(p<0.001)$ or 12 months $(p<0.001)$. The IKDC score was $41.7 \pm$ 9.2 before treatment and was $67.3 \pm 14.1$ at 12 months $(\mathrm{p}<0.001)$. A better symptomatic relief was observed in patients with early knee OA (stage 1 and 2 of Kellgren-Lawrence classification).

Conclusions: This study suggests that leukocyte-poor-PRP injections are effective in reducing pain symptoms and improving functional outcomes in patients with knee OA at short-term follow-up.
\end{abstract}

Keywords: Knee; Osteoarthritis; Platelet-rich-plasma; Pain; Conservative Treatment

\section{Introduction}

The increasing aging of the population and the peculiar characteristics of articular cartilage are the main reasons for the widespread of the knee osteoarthritis (OA) disease worldwide [1]. The current most complete and long-lasting treatment for knee $\mathrm{OA}$ is a total knee arthroplasty surgery, which is characterized by excel- lent functional results. However, it should be considered that $15 \%$ of patients undergoing to knee arthroplasty had a severe residual pain [2].

In order to control symptoms and improve quality of life, several alternative treatments have been proposed, such as physiokinesis therapy, use of analgesic, changes in lifestyle and eating habits and 
joint injections. In this context, platelet-rich plasma (PRP) is now routinely used for the symptomatic treatment of $\mathrm{OA}$ with good results in the short and medium period. The use of this procedure is justified by a good safety profile, ease of use and production, and low management costs [3]. The efficacy of PRP for the management of knee $\mathrm{OA}$ is still controversial and the superiority of one technique over another is questioned. In a recent meta-analysis of randomized controlled clinical trials the use of PRP, in particular leukocyte-poor PRP, was associated with improved clinical outcomes compared to hyaluronic acid [4]. Similarly, a network meta-analysis of 30 randomized clinical trials showed that PRP injections had the best clinical outcome compared to steroids, hyaluronic acid and placebo for patients with knee OA [5]. Due to the heterogeneity of the included studies and their relatively small simple size, the optimum treatment for knee OA is still inconclusive.

In our study, we reported the efficacy in term of pain reduction and knee function of leukocyte-poor PRP (Lp-PRP) injections in a large number of patients with symptomatic knee OA.

\section{Materials and Methods}

This is a retrospective, single center observational study carried out from 2013 to 2018. Patients with symptomatic knee OA who underwent to injective therapy with Lp-PRP were included in the study. Exclusion criteria were: previous knee surgeries, use of other symptomatic/injective treatments or physical therapy in the previous 6 months, active infections, knee pain for less than 3 months from the inclusion in the study, post-traumatic knee pain, positive history for epilepsy or severe cerebrovascular disease, immunosuppressive drugs, or treatment with anticoagulants or antiaggregants, haemoglobin value $<11 \mathrm{~g} /$ decilitre and platelet count $<150,000 / \mathrm{mmc}$.

After excluded 51 patients according to the study criteria we enrolled a total of 213 patients.

Patients were treated with one injection every 14 days for a total of three procedures. The injection was performed in sterile conditions through a free-handed technique and a lateral suprapatellar approach with a 22-gauge needle. After the injection, patients were sent home with instructions to restrict the use of the leg for 48 hours and to use cold therapy/ice on the affected area to relieve pain. All the procedures were performed by seven orthopedics with at least 5-years experience.
Before the treatment, all patients were classified using KellgrenLawrence grading scale by evaluating antero-posterior X-ray of the knee. Stage 1 and 2 referred to early knee OA, stage 3 to moderate $\mathrm{OA}$, stage 4 to severe $\mathrm{OA}$. Patients were evaluated the time of study inclusion and at 3,6 and 12 months by phone contact or clinical visit. During follow-up, the following evaluation tools were estimated: the VAS score and the IKDC Subjective Knee Form. The need of pain medication for the knee, patient satisfaction and any adverse events were also reported. Collection of data was retrospectively performed. The study was approved by the local institutional review boards.

\section{PRP preparation method}

The conditioned plasma preparation kit was used for PRP preparation. Gel preparation was performed according to Italian society transfusion medicine and immune-haematology (SIMTI) recommendations [6].

Preparation of anticoagulated blood with Citrate-PhosphateDestrose plus $0.25 \mathrm{mM}$ adenine (CPDA-1).

Content of $1,000,000 \pm 20 \%$ Platelet per micro-litre (effective dose).

For the preparation of the product a venous blood sample was taken with Terumo blood-bag containing sodium citrate (CPDA-1) as anticoagulant. The amount of blood to be taken, which varies according to the basal platelet concentration of the patient, varies from 100 to $150 \mathrm{~mL}$. The product obtained was then subjected to centrifugation at low rpm (1000 rpm for 20') in order to effectively separate the platelets without lysing them. After the first centrifugation, a second centrifugation is performed at higher rpm (2500 rpm for 20'). At this point the platelet-poor supernatant plasma part is eliminated and the platelet-rich and leukocyte-poor plasma is collected. In case of mono-lateral infiltration a volume of PRP of $9 \mathrm{~mL}$ is collected, which becomes $18 \mathrm{~mL}$ in case of bilateral procedures. The produced aliquots ( $3 \mathrm{~mL}$ per single dose) are stored at $-40^{\circ} \mathrm{C}$ and thawed on the day of the procedure.

\section{Statistical analyses}

Patient's characteristics were summarized as mean \pm standard deviation (SD) for continuous variables and as absolute numbers and percentages for categorical variables. We compared categorical variables using the $\chi^{2}$ test and continuous variables with the MannWhitney $\mathrm{U}$ test. A two-sided $\mathrm{P}<0.05$ was considered significant. 
One-way ANOVA was performed to assess differences between groups when the Levene test for homogeneity of variances was not significant $(\mathrm{p}<0.05)$. The following subgroup analyses were also performed: i) one cycle versus two or more cycles, ii) according to Kellgren-Lawrence grading scale, iii) according to age.

All statistical analyses were performed using the IBM SPSS Statistics version 23.0 (IBM Corporation, Armonk, NY).

\section{Results}

Mean age was $68.1 \pm 9.7$ years and $59.6 \%$ were female. The majority of patients $(74.2 \%)$ had comorbidities: hypertension (43.7\%), hypercholesterolemia (8.9\%) and anxiety/depressive syndrome (5.2\%). Main clinical features of the patients included are reported in table 1 .

\begin{tabular}{|l|c|}
\hline Age, mean $\mathbf{\text { SD }}$ & Patients included (213 pts) \\
\hline Female, n (\%) & $68.1 \pm 9.7(37-92)$ \\
\hline Comorbidities, n (\%) & $127(59.6)$ \\
\hline Hypertension, n (\%) & $158(74.2)$ \\
Hypercholesterolemia, n (\%) & $93(43.7)$ \\
BMI* $\geq 28$, n (\%) & $19(8.9)$ \\
Previous cancer, n (\%) & $10(4.7)$ \\
Anxiety-depression, n (\%) & $7(3.3)$ \\
\hline Treatment cycles, mean \pm SD & $11(5.2)$ \\
\hline Monolateral treatment, n (\%) & $2.4 \pm 1.9(1-11)$ \\
\hline
\end{tabular}

Table 1: Main clinical features of patients included.

$$
\text { *Body mass index. }
$$

Ninety-four patients (44.1\%) performed a single cycle of infiltration and the mean number of infiltration cycles was $2.4 \pm 1.9$. One-hundred patients were affected by unilateral OA while the other 113 presented bilateral OA, thus making a total of 326 knees treated.

The mean VAS score before treatment was $6.5 \pm 1.4$ (range from 2 to 10 ), $1.6 \pm 1.6$ at 3 months, $1.4 \pm 1.5$ at 6 months and $1.7 \pm 1.7$ at 12 months (Table 2). A significant reduction was observed between the VAS score pre-treatment and the VAS score at 3 months $(\mathrm{p}<0.001), 6$ months $(\mathrm{p}<0.001)$ and 12 months $(\mathrm{p}<0.001)$. The IKDC score before the treatment was $41.7 \pm 9.2$ and at 12 months was $67.3 \pm 14.1(\mathrm{p}<0.001)$.
At 12 months, adverse events were reported in $9.4 \%$ of patients (Table 2). The most common adverse events were peri-procedural pain (5.2\%), joint effusion (3.4\%) and transient limp (1\%). Sixtyseven percent of patients referred to be satisfied at 12 months and a clinical improvement was reported after a mean of $2.3 \pm 0.7$ infiltrations.

\begin{tabular}{|l|c|}
\hline & $\begin{array}{c}\text { Patients included (213 } \\
\text { pts) }\end{array}$ \\
\hline VAS pre-treatment, mean \pm SD & $6.5 \pm 1.4(2-10)$ \\
\hline VAS 3-months, mean \pm SD & $1.6 \pm 1.6(0-8)$ \\
\hline VAS 6-months, mean \pm SD & $1.4 \pm 1.5(0-8)$ \\
\hline VAS 12-months, mean \pm SD & $1.7 \pm 1.7(0-8)$ \\
\hline IKDC pre-treatment, mean \pm SD & $41.7 \pm 9.2$ \\
\hline IKDC 12-months, mean \pm SD & $67.3 \pm 14.1$ \\
\hline Adverse events at 12-months, $\mathrm{n}(\%)$ & $20(9.4)$ \\
Pain, $\mathrm{n}(\%)$ & $11(5.2)$ \\
Joint effusion, $\mathrm{n}(\%)$ & $7(3.4)$ \\
Limp, $\mathrm{n}(\%)$ & $2(1.0)$ \\
\hline Analgesic effect at 12 -months, $\mathrm{n}(\%)$ & $143(67.1)$ \\
\hline
\end{tabular}

Table 2: Main study outcomes.

\section{Subgroup analyses}

Among patients undergoing to one cycle of infiltration compared to patients undergoing to more than one cycle, no significant differences were observed in term of VAS scale score pre-treatment and during follow-up (Table 3).

\begin{tabular}{|l|c|c|c|}
\hline & $\begin{array}{c}\text { 1 cycle 94 } \\
\text { pts }\end{array}$ & $\begin{array}{c}\text { 2 or more } \\
\text { cycles 119 pts }\end{array}$ & P-value \\
\hline VAS pre-treatment & $6.7 \pm 1.5$ & $6.4 \pm 1.3$ & 0.075 \\
\hline VAS 3-months & $1.6 \pm 1.7$ & $1.6 \pm 1.5$ & 0.804 \\
\hline VAS 6-months & $1.4 \pm 1.6$ & $1.3 \pm 1.5$ & 0.570 \\
\hline VAS 12-months & $1.8 \pm 1.8$ & $1.7 \pm 1.7$ & 0.589 \\
\hline
\end{tabular}

Table 3: Comparison between patients underwent 1 cycle of infiltration Vs. patients underwent more than one cycle.

When evaluating the outcome according to the Kellgren-Lawrence classification, we observed a significant VAS scale score reduction and a significant IKDC score improving in patients with early knee OA (stage 1 and 2) compared to moderate and severe knee OA (stage 3 and 4) (Table 4). Finally, no significant differences in term of VAS scale score and IKDC score were observed in patients at different age (Table 5). 
The Effects of Leukocyte-Poor Platelet Rich Plasma for the Symptomatic Knee Osteoarthritis: A Single Centre Experience

\begin{tabular}{|l|c|c|c|c|c|}
\hline & Stage 1 13 pts & Stage 2 131 pts & Stage 3 44 pts & Stage 4 25 pts & P for comparison \\
\hline VAS pre-treatment & $5.8 \pm 1.9$ & $6.6 \pm 1.3$ & $6.6 \pm 1.4$ & $6.4 \pm 1.4$ & 0.229 \\
\hline VAS 3-months & $1.0 \pm 0.9$ & $1.3 \pm 1.4$ & $1.8 \pm 1.5$ & $3.3 \pm 2.4$ & $<0.001$ \\
\hline VAS 6-months & $0.9 \pm 0.7$ & $1.1 \pm 1.2$ & $1.4 \pm 1.4$ & $3.1 \pm 2.2$ & $<0.001$ \\
\hline VAS 12-months & $1.1 \pm 0.9$ & $1.4 \pm 1.3$ & $1.7 \pm 1.7$ & $3.5 \pm 2.5$ & $<0.001$ \\
\hline IKDC pre-treatment & $46.9 \pm 9.9$ & $43.7 \pm 8.5$ & $38.5 \pm 8.9$ & $34.7 \pm 7.9$ & $<0.001$ \\
\hline IKDC 12-months & $74.5 \pm 6.2$ & $72.9 \pm 8.7$ & $61.5 \pm 13.5$ & $45.3 \pm 14.4$ & $<0.001$ \\
\hline
\end{tabular}

Table 4: Study outcomes according to Kellegren-Lawrence scale.

\begin{tabular}{|l|c|c|c|c|c|}
\hline & Age $<\mathbf{5 0} 9$ pts & Age 50-65 60 pts & Age 66-75 96 pts & Age >75 48 pts & P for comparison \\
\hline VAS pre-treatment & $6.7 \pm 1.0$ & $6.6 \pm 1.4$ & $6.4 \pm 1.4$ & $6.5 \pm 1.4$ & 0.803 \\
\hline VAS 3-months & $0.7 \pm 0.9$ & $1.9 \pm 1.6$ & $1.4 \pm 1.6$ & $1.8 \pm 1.6$ & 0.357 \\
\hline VAS 6-months & $0.7 \pm 0.9$ & $1.5 \pm 1.6$ & $1.3 \pm 1.5$ & $1.4 \pm 1.4$ & 0.460 \\
\hline VAS 12-months & $0.8 \pm 1.0$ & $1.9 \pm 1.9$ & $1.6 \pm 1.7$ & $1.8 \pm 1.7$ & 0.334 \\
\hline IKDC pre-treatment & $46.4 \pm 9.2$ & $40.5 \pm 8.5$ & $42.4 \pm 9.3$ & $41.1 \pm 9.9$ & 0.253 \\
\hline IKDC 12-months & $76.0 \pm 6.4$ & $66.4 \pm 14.0$ & $67.7 \pm 13.9$ & $65.8 \pm 15.3$ & 0.232 \\
\hline
\end{tabular}

Table 5: Study outcomes according to age.

\section{Discussion}

In our study of 213 patients (a total of 326 knees treated), we found that the use Lp-PRP injections significantly reduced pain symptoms and improved quality of life in patients with knee OA at 12 months. Patients with early knee OA had the best functional and clinical results after Lp-PRP injections.

The use of PRP in the treatment of symptomatic knee OA is considered safe and effective [1], although there is no conclusive evidence yet. Methodological errors and incomplete literature are the basis for conflicting results. One of the causes is the heterogeneity of PRP preparation and injection techniques [7]. Some authors use Lr-PRP while others use Lp-PRP. Several studies show that the presence of a high number of leukocytes in Lr-PRP is related to a higher incidence of side effects related to the pro-inflammatory potential of leukocytes [8-11].

On the other hand, Cavallo., et al. [12], have shown that in vitro the production of hyaluronic acid and the proliferation of chondrocytes was higher following the administration of Lr-PRP. Finally, a recent meta-analysis of 18 randomized clinical trials shows that the efficacy of Lr-PRP was associated with significantly better Subjective IKDC scores compared to leukocyte-rich PRP [4].

Another variable to consider is the number of platelets in PRP, which will have an effect on the knee OA. It could be hypothesized that methods that involve a single centrifugation, and therefore fewer platelets in the final product, are less effective than double centrifugation preparation techniques that guarantee a higher platelet concentration. It is not yet clear what is the optimal concentration to obtain the maximum pain-relieving action [13]. On the contrary, what is really fundamental is the state of activity of the platelets and the release of platelet factors [14].

Focusing on the injection procedure, many heterogeneous aspects emerge in literature: the infiltrative volumes used $(3 / 8 \mathrm{~mL})$, the number of injection $(1 / 3)$, the interval between them $(1 / 2 / 3$ weeks), storage aspects as well as activation methods vary widely between authors. Kavadar., et al. [15] reported that PRP is an effective and reliable treatment for functional status and pain for stage 3 of knee OA and a minimum of two injections appears to be appropriate. Jubert., et al. [16] showed that a single PRP intra-articular injection is effective for relieving pain and improving activities of daily living and quality of life in late-stage knee OA. In our centre, we performed one injection every 15 days for a total of three procedures, regardless of the knee OA-stage.

PRP storage is also crucial aspect: freeze-thawing allows easier patient management, but could impair platelet function and lifespan, and could increase the risk of infections [17]. Roffi., et al. [18] concluded that cryopreservation is a safe procedure, which suffi- 
ciently preserves PRP quality and its biological activity. Ours is a high-volume center so freeze-thawing makes the procedure easier and faster; moreover we have never recorded any superficial or deep infections.

Even the method of intra-articular infiltration could affect the outcome of the treatment: the access used at the time of infiltration could increase the patient's periprocedural pain; even more frequently it could cause inoculation of the preparation in the adipose body of Hoffa, especially with the antero-medial and anterolateral access, causing a phlogistic process in the hours following the procedure, as well as an alteration of the microenvironment of action of the platelet microgranules. An effective alternative, but time-consuming, could be the use of eco-guided techniques that ensure the inoculation of the PRP within the joint space, guaranteeing a more effective treatment and limiting the possible causes of pain $[19,20]$.

In such a heterogeneous scientific contest, our study aims to clarify some aspects: the use of PRP in our center provides a method validated and standardized over 11 years of clinical experience. The strengths of this observational study are the not negligible sample size, the strict exclusion criteria, the standardization in the preparation and infiltration procedure and a systematic follow-up at 3, 6 and 12 months. All patients were also classified with standard X-rays and divided into groups. PRP preparation technique in this study was standardized by our transfusion medicine department and no commercial filters were used.

The limitations of the study are mainly represented by a nonhomogeneous sample of patients due to the high prevalence of stages 2 and 3 according to Kellgren-Lawrence classification and the number of cycles, and the lack of a control group. Another limitation to consider is the use of non-echo-guided injection technique with a potential risk of extra-articular inoculation; this could explain a peri-procedural pain rate of $5.2 \%$ that should be remarked. However, it is a transient pain that resolves spontaneously within 48 hours.

Our results confirmed that Lp-PRP is effective in pain control in each stage of knee OA, although stages 1 and 2 sec. KellgrenLawrence have better functional and clinical results. Lp-PRP may provide short-term clinical benefit up to 12 months. The effect is not influenced by the age of the patients.

\section{Conclusion}

In conclusion, Lp-PRP injections are a safe and effective, conservative approach in patients with knee $\mathrm{OA}$. A better symptomatic relief was observed in patients with early knee OA. The effect seems to be not influenced by the age of the patients.

\section{Funding}

Authors disclose no funding sources.

\section{Conflicts of Interests}

The authors declare that they have no conflict of interest.

\section{Bibliography}

1. Xing D., et al. "Intra-articular platelet-rich plasma injections for knee osteoarthritis: An overview of systematic reviews and risk of bias considerations". International Journal of Rheumatic Diseases 20.11 (2017): 1612-1630.

2. Wylde V., et al. "Persistent pain after joint replacement: prevalence, sensory qualities, and postoperative determinants". Pain 152.3 (2011): 566-572.

3. Bennell KL., et al. "Platelet-Rich Plasma for the Management of Hip and Knee Osteoarthritis". Current Rheumatology Reports 19.5 (2017): 24.

4. Belk JW., et al. "Platelet-Rich Plasma Versus Hyaluronic Acid for Knee Osteoarthritis: A Systematic Review and Meta-analysis of Randomized Controlled Trials". American Journal of Sports Medicine (2020).

5. Migliorini F., et al. "Comparison between intra-articular infiltrations of placebo, steroids, hyaluronic and PRP for knee osteoarthritis: a Bayesian network meta-analysis [published online ahead of print, 2020 Jul 28]". Archives of Orthopaedic and Trauma Surgery (2020).

6. Liumbruno G., et al. "Italian Society of Transfusion Medicine and Immunohaematology (SIMTI) Work Group. Recommendations for the transfusion of plasma and platelets". Blood Transfus 7.2 (2009): 132-150.

7. Laver L., et al. "PRP for Degenerative Cartilage Disease: A Systematic Review of Clinical Studies". Cartilage 8.4 (2017): 341364.

8. Pifer MA., et al. "Matrix metalloproteinase content and activity in low-platelet, low-leukocyte and high-platelet, high-leukocyte platelet rich plasma (PRP) and the biologic response to PRP by human ligament fibroblasts". American Journal of Sports Medicine 42.5 (2014): 1211-1218. 
9. Braun HJ., et al. "The effect of platelet-rich plasma formulations and blood products on human synoviocytes: implications for intra-articular injury and therapy". American Journal of Sports Medicine 42.5 (2014): 1204-1210.

10. Dragoo JL., et al. "Comparison of the acute inflammatory response of two commercial platelet-rich plasma systems in healthy rabbit tendons". American Journal of Sports Medicine 40.6 (2012): 1274-1281.

11. Filardo G., et al. "Platelet-rich plasma intra-articular injections for cartilage degeneration and osteoarthritis: single- versus double-spinning approach". Knee Surgery, Sports Traumatology, Arthroscopy 20.10 (2012): 2082-2091.

12. Cavallo C., et al. "Comparison of platelet-rich plasma formulations for cartilage healing: an In vitro study". Journal of Bone and Joint Surgery American 96.5 (2014): 423-429.

13. Kuffler DP. "Variables affecting the potential efficacy of PRP in providing chronic pain relief". Journal of Pain Research 12 (2018): 109-116.

14. Perut F., et al. "Preparation method and growth factor content of platelet concentrate influence the osteogenic differentiation of bone marrow stromal cells". Cytotherapy 15.7 (2013): 830-839.

15. Kavadar G., et al. "Effectiveness of platelet-rich plasma in the treatment of moderate knee osteoarthritis: a randomized prospective study". Journal of Physical Therapy Science 27.12 (2015): 3863-3867.

16. Joshi Jubert N., et al. "Platelet-Rich Plasma Injections for Advanced Knee Osteoarthritis: A Prospective, Randomized, Double-Blinded Clinical Trial". Orthopaedic Society for Sports Medicine 5.2 (2017): 2325967116689386.

17. Kon E., et al. "PRP for the treatment of cartilage pathology". The Open Orthopaedics Journal 7 (2013): 120-128.

18. Roffi A., et al. "Does platelet-rich plasma freeze-thawing influence growth factor release and their effects on chondrocytes and synoviocytes?" BioMed Research International (2014): 692913.

19. Sibbitt WL JR., et al. "Does ultrasound guidance improve the outcomes of arthrocentesis and corticosteroid injection of the knee?" Scandinavian Journal of Rheumatology 41.1 (2012): 66-72.

20. Berkoff DJ., et al. "Clinical utility of ultrasound guidance for intra-articular knee injections: a review". Clinical Interventions in Aging 7 (2012): 89-95.

\section{Assets from publication with us}

- Prompt Acknowledgement after receiving the article

- Thorough Double blinded peer review

- Rapid Publication

- Issue of Publication Certificate

- High visibility of your Published work

Website: https://www.actascientific.com/

Submit Article: https://www.actascientific.com/submission.php Email us: editor@actascientific.com

Contact us: +919182824667 